\title{
An Anatomical Variation in the Cervical Carotid Artery of a Young Stroke Patient
}

\author{
Tatsunori Natori, Shinsuke Narumi, Takafumi Suzuki, Mitsunobu Sato, Keisuke Tsuda, \\ Asami Kamada, Makiko Yoshida, Kiyotaka Oi, Yoshio Suzuki and Yasuo Terayama
}

\begin{abstract}
:
The cervical carotid artery has been reported to show anatomical variations. We report the case of a young stroke patient with a small right-parietal-lobe infarction whose cervical carotid artery showed anatomical variation. The right internal carotid artery (ICA) originated at the C2 level of the external carotid artery with protrusion at the right carotid bifurcation. The vessel wall of the protrusion showed a high signal intensity on T1-weighted magnetic resonance carotid plaque imaging. The protrusion, considered a remnant of the ICA, possibly caused the stroke due to the formation of thrombi as a result of changes in blood flow and viscosity.
\end{abstract}

Key words: stroke, non-bifurcating artery

(Intern Med 58: 123-126, 2019)

(DOI: 10.2169/internalmedicine.1526-18)

\section{Introduction}

Several anatomical variations of the cervical carotid artery have been found, especially in the carotid bifurcation (1-5). However, these variations have been considered as cervical carotid artery anomalies and have not implicated in ischemic or hemorrhagic stroke. We herein report a very rare case of an anatomical variation of the cervical carotid artery that was considered to be the cause of an ischemic stroke in a young patient.

\section{Case Report}

A 47-year-old man presented to our emergency department 4 hours after a sudden onset of left hand weakness and dysarthria. However, the symptoms disappeared within a few minutes of the onset, and he was asymptomatic when he visited our hospital. He had no relevant familial or medical history. Magnetic resonance (MR) imaging revealed no evidence of ischemic or hemorrhagic stroke. Thus, the patient was diagnosed with a transient ischemic attack and was given aspirin (100 mg).

One month later, he revisited our hospital 2 hours after the onset of left hand dysesthesia. A neurological examina- tion showed slight weakness and dysesthesia in his left hand. The National Institute of Health Stroke Scale score was 1. Diffusion-weighted imaging showed two small highintensity lesions on the right parietal lobe (Fig. 1A). MR angiography (MRA) revealed no significant stenosis of the intracranial arteries (Fig. 1B), and there were no abnormal changes (e.g., old lacuna infarction or old hemorrhage) on T2-weighted imaging. Moreover, 24-h electrocardiogram (ECG) monitoring for 19 days showed a normal sinus rhythm and did not detect paroxysmal arterial fibrillation or any other arrhythmias. A transthoracic echocardiogram and transesophageal echocardiogram revealed no intracardiac thrombi and a patent foramen ovale. A blood analysis revealed abnormal uric acid $(7.3 \mathrm{mg} / \mathrm{dL})$ and triglyceride (364 $\mathrm{mg} / \mathrm{dL}$ ) levels. The blood count and coagulation state, including the levels of proteins $\mathrm{C}$ and $\mathrm{S}$, were normal. There was no inflammation suggestive of vasculitis. On digital subtraction angiography, the right internal carotid artery (ICA) was shown to originate from the $\mathrm{C} 2$ level of the right external carotid artery (ECA), and a protrusion was observed near the right carotid bifurcation (Fig. 2A and B). The ECA branch, superior thyroid artery (TA), lingual artery (LA), facial artery (FA), and occipital artery (OA) originated from the ECA. The left common carotid artery (CCA), ICA, ECA, bilateral vertebral artery, and basilar artery were nor-

Department of Neurology and Gerontology, Institute for Biomedical Sciences, Iwate Medical University, Japan 

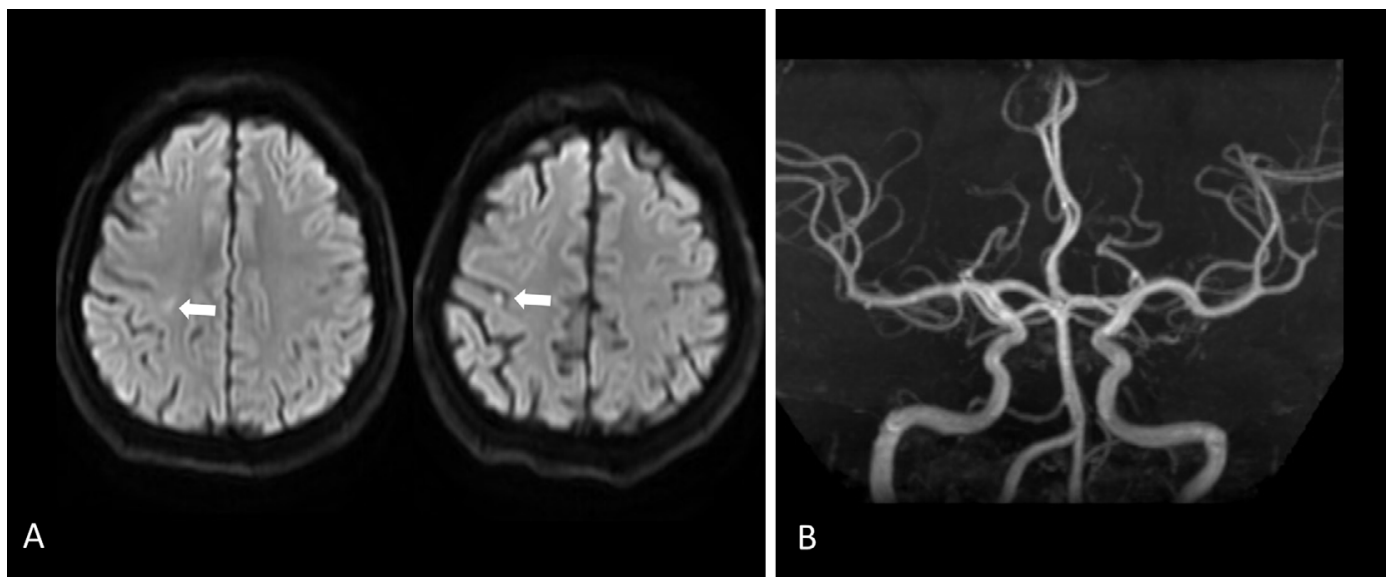

Figure 1. Diffusion-weighted imaging (DWI) (A) and three-dimensional time-of-flight magnetic resonance angiography (MRA) (B). Lesions with a high signal intensity were observed in the parietal lobe on DWI (A, arrow). MRA revealed no significant stenosis in the intracranial arteries (B).
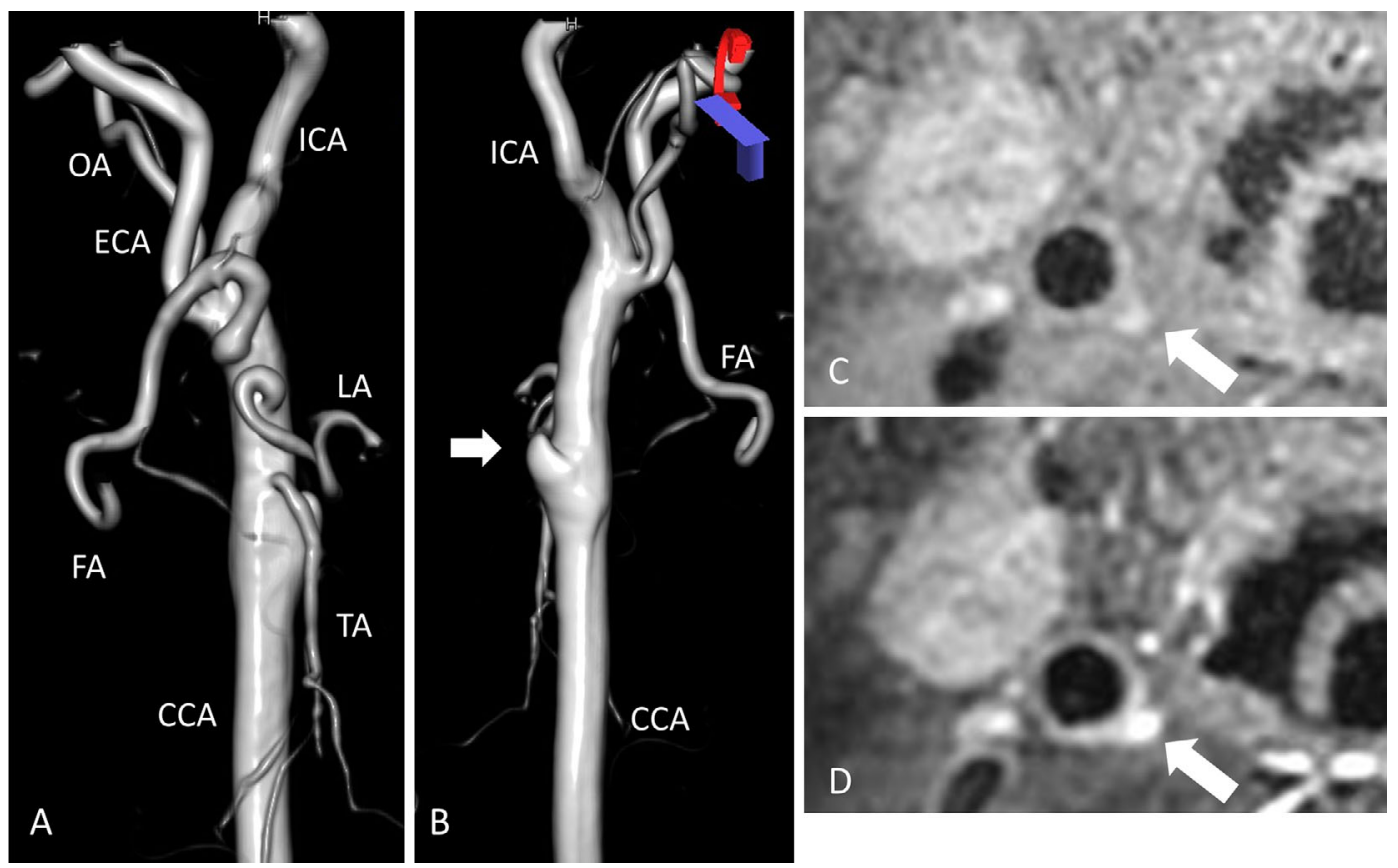

Figure 2. Digital subtraction angiography (A, B) and T1-weighted imaging (T1WI) obtained by three-dimensional (3D) magnetic resonance (MR) carotid plaque imaging $(C, D)$. The right internal carotid artery (ICA) originated from the $\mathrm{C} 2$ level of the right external carotid artery (ECA), while a protrusion was observed at the right carotid bifurcation. The branch of the right ECA, superior thyroid artery (TA), lingual artery (LA), facial artery (FA), and occipital artery (OA) originated from the right ECA (A, B). The protrusion showed a high signal intensity on T1W MR carotid plaque imaging $(C$, arrow) and was enhanced on post-contrast T1WI (D, arrow). These results suggest the presence of thrombi including a hemorrhagic component.

mal. No atherosclerotic changes were found in the intracranial or cervical carotid arteries. The protrusion showed high intensity and enhancement on a T1-weighted image (T1W) from three-dimensional (3D) MR carotid plaque imaging, suggesting the presence of thrombi, including a hemorrhagic component (Fig. 2C and D). Two-dimensional (2D) carotid ultrasonography (US) revealed a disturbed flow in the protrusion at the right carotid bifurcation (Fig. 3).

An examination suggested that the patient's stroke was caused by an artery-to-artery embolism due to the protrusion at the right carotid bifurcation. The patient was given clopidogrel $(75 \mathrm{mg} /$ day) to prevent another stroke. His symptoms disappeared 2 days after the onset of stroke, and he was discharged 19 days after admission. At present, there has been no recurrence of stroke for more than 3 years. In addition, ECG has been performed several times since the patient's discharge, and neither paroxysmal arterial fibrillation nor any other arrhythmias have been observed. 

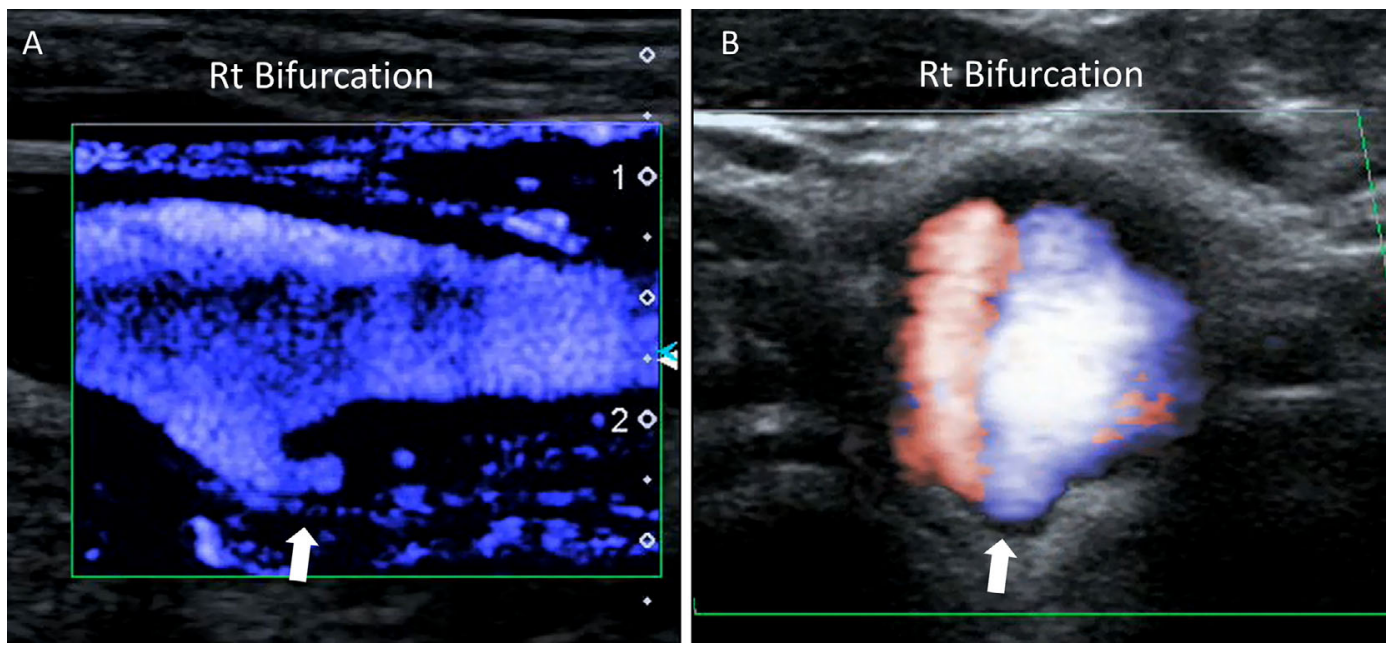

Figure 3. Two-dimensional carotid ultrasonography (A, B). Disturbed flow was seen in the protrusion at the right carotid bifurcation ( $A$ and $B$, arrow).

\section{Discussion}

There have been several reports on rare anatomical variations of the cervical carotid artery (1-5). These abnormalities were considered to be congenital and to develop during angiogenesis $(6,7)$; furthermore, they have been considered to be incidental and were not known to cause stroke. The present case represents a very rare case of an anatomical variation in the carotid bifurcation and internal carotid artery that resulted in ischemic stroke. The anatomical variation of the present case is considered a variant of the nonbifurcating cervical carotid artery. A non-bifurcating artery may be caused by segmental agenesis of the proximal ICA, causing the major branches of the proximal ECA to develop separately from the terminal segment of the CCA or proximal ICA (8-13). In the present case, the protrusion was located in the carotid bifurcation at the level of the $\mathrm{C} 4 / 5$ vertebra, where the normal ICA originated. Thus, the protrusion was considered to be a remnant of the ICA.

While there are several reports on non-bifurcating arteries, there is only one reported case of a non-bifurcating artery with protrusion of a remnant ICA (14). This is the second report of this anatomical variation, but it is the first report to describe this anatomical variation as a cause of stroke. Disturbed flow was detected in the protrusion by US. In addition, a high signal intensity and the wall enhancement on MR carotid plaque imaging suggested the presence of thrombi, including a hemorrhagic component, which are also seen in unstable plaque $(15,16)$. These results suggest that the protrusion caused stroke because of the change in blood flow and viscosity, leading to the formation of thrombi.

The present case is associated with several limitations. First, it is difficult to prove the presence of thrombi in the protrusion because of the imaging findings were not correlated with the histopathological findings in the present case. In addition, no temporal changes could be detected in the findings because MRI of the plaque was not performed on an outpatient basis, while computed tomography (CT) angiography and US were performed. However, according to previous studies, the high signal intensity on T1-weighted MR carotid plaque imaging, which is considered to be the least invasive method for evaluating plaque components, suggested the presence of thrombi, including a hemorrhagic component $(14,15)$. Second, all appropriate examinations for detecting the embolic sources of stroke in this patient were performed, with the exception of an insertable cardiac monitor (ICM), which had not yet been introduced and which was not available to us.

In summary, we herein described a very rare case of an anatomical variation in the cervical carotid artery. This abnormality appeared to be a subtype of the non-bifurcating cervical carotid artery. In addition, the protrusion, which was considered to be a remnant of ICA, was strongly suggested as the cause of the stroke.

The authors state that they have no Conflict of Interest (COI).

\section{References}

1. Ueda S, Kohyama Y, Takase K. Peripheral hypoglossal nerve palsy caused by lateral position of the external carotid artery and an abnormally high position of bifurcation of the external and internal carotid arteries-a case report. Stroke 15: 736-739, 1984.

2. Ito H, Mataga I, Kageyama I, Kobayashi K. Clinical anatomy in the neck region-the position of external and internal carotid arteries may be reversed-. Okajimas Folia Anat Jpn 82: 157-168, 2006.

3. Uchino A, Sawada A, Hirakawa N, Totoki T, Kudo S. Congenital absence of the internal carotid diagnosed during investigation of trigeminal neuralgia. Eur Radiol 12: 2339-2342, 2002.

4. Moon WJ, Porto L, Lanfermann H, Weis R, Zanella FE. Agenesis of internal carotid artery associated with congenital anterior hypopituitarism. Neuroradiology 44: 138-142, 2002.

5. Michalinos A, Chatzimarkos M, Arkadopoulos N, Troupis T. Anatomical considerations on surgical anatomy of the carotid bifurcation. Anat Res Int 2016: 8, 2016. 
6. Lasjaunias P. Segmental identity and vulnerability in cerebral arteries. Interventional Neuroradiology 6: 113-124, 2000.

7. Gaillound P, Clatterbuck RE, Fasel JH, Tamargo RJ, Murphy KJ. Segmental agenesis of the internal carotid artery distal to the posterior communicating artery leading to the definition of a new embryokogic segment. AJNR Am J Neuroradiol 25: 1189-1193, 2004.

8. Morimoto T, Nitta K, Kazekawa K, Hashizume K. The anomaly of a non-bifurcating cervical carotid artery. Case report. J Neurosurg 72: 130-132, 1990.

9. Ooigawa H, Nawashiro H, Fukui S, et al. Non-bifurcating cervical carotid artery. J Clin Neurosci 13: 944-947, 2006.

10. Kiyose $H$, Mori $H$, Tanoue $S$, et al. Non-bifurcating carotid artery coexisting with transverse sinus dural arteriovenous fistula. Neuroradiology 51: 697-698, 2009

11. Uchino A, Saito N, Watadani T, Mizukoshi W, Nakajima R. Nonbifurcating cervical carotid artery diagnosed by MR angiography. AJNR Am J Neuroradiol 32: 1119-1122, 2011.

12. Uchino A, Saito N, Watadani T. Congenital external carotidinternal carotid artery anastomosis diagnosed by MR angiography J Neuroimaging 23: 96-97, 2013.
13. Lourenco P, Heran M. Asymptomatic non-bifurcating carotid artery. A case report and literature review. Neuroradiol J 27: 393396, 2014

14. Sasaki T, Nagashima H, Oya F, Satoh D, Kobayashi S. Carotid artery stenting for atheroscletotic stenosis associated with nonbifurcating cervical carotid artery -case report and embryological considerations-. Neurol Med Chir (Tokyo) 53: 228-232, 2013.

15. Narumi S, Sasaki M, Ohba H, et al. Predicting carotid plaque characteristics using quantitative color-coded T1-weighted MR plaque imaging: correlation with carotid endarterectomy specimens. AJNR Am Neuroradiol 35: 766-771, 2014.

16. Natori T, Sasaki M, Miyoshi M, et al. Intracranial plaque characterization in patients with acute ischemic stroke using pre- and post- contrast three-dimensional magnetic resonance vessel wall imaging. J Stroke Cerebrovasc Dis 25: 1425-1430, 2016.

The Internal Medicine is an Open Access journal distributed under the Creative Commons Attribution-NonCommercial-NoDerivatives 4.0 International License. To view the details of this license, please visit (https://creativecommons.org/licenses/ by-nc-nd/4.0/).

(C) 2019 The Japanese Society of Internal Medicine Intern Med 58: 123-126, 2019 